\title{
Cryptococcosis in HIV-negative Patients with Renal Dialysis: A Retrospective Analysis of Pooled Cases
}

\author{
Nan Hong • Min Chen · Wenjie Fang • Abdullah M. S. Al-Hatmi • \\ Teun Boekhout $\cdot$ Jianping Xu $\cdot$ Lei Zhang $\cdot$ Jia Liu $\cdot$ Weihua Pan • \\ Wanqing Liao
}

Received: 31 March 2017/Accepted: 13 June 2017/Published online: 30 June 2017

(C) The Author(s) 2017. This article is an open access publication

\begin{abstract}
Cryptococcosis is a lethal fungal infection mainly caused by Cryptococcus neoformans/C. gattii species. Currently, our understanding of cryptococcosis episodes in HIV-negative patients during renal dialysis remains scarce and fragmented. Here, we performed an analysis of pooled cases to systemically summarize the epidemiology and clinical characteristics of cryptococcosis among HIV-negative patients with renal dialysis. Using pooled data from our hospital and studies identified in four medical databases, 18 cases were identified and analyzed. The median duration time of renal dialysis for peritoneal renal dialysis and hemodialysis cases was 8 months and 36 months, respectively. Several nonneoformans/gattii species were identified among the
\end{abstract}

Nan Hong, Min Chen and Wenjie Fang contributed equally to this work and shared the first authorship.

N. Hong · M. Chen · W. Fang $\cdot$ L. Zhang

J. Liu · W. Pan $(\bowtie) \cdot$ W. Liao $(\square)$

Shanghai Key Laboratory of Molecular Medical

Mycology, Department of Dermatology, Changzheng

Hospital, Second Military Medical University, Shanghai,

China

e-mail: panweihua@medmail.com.cn

W. Liao

e-mail: liaowanqing@sohu.com

J. Xu

Department of Biology, McMaster University, Hamilton, Canada renal dialysis recipients with cryptococcosis, particularly Cryptococcus laurentii and Cryptococcus albi$d u s$, which share similar clinical manifestations as those caused by $C$. neoformans and $C$. gattii. Our analyses suggest that physicians should consider the possibility of the occurrence of cryptococcosis among renal dialysis recipients even when cryptococcal antigen test result is negative. The timely removal of the catheter is crucial for peritoneal dialysis patients with cryptococcosis. In addition, there is a need for optimized antifungal treatment strategy in renal dialysis recipients with cryptococcal infections.

Keywords Cryptococcosis $\cdot$ Renal dialysis $\cdot$ Pooled analysis

\author{
A. M. S. Al-Hatmi - T. Boekhout \\ Westerdijk Fungal Biodiversity Institute, Utrecht, \\ The Netherlands \\ T. Boekhout \\ Institute of Biodiversity and Ecosystem Dynamics \\ (IBED), University of Amsterdam, Amsterdam, \\ The Netherlands \\ A. M. S. Al-Hatmi \\ Directorate General of Health Services, Ministry of \\ Health, Ibri Hospital, Ibri, Oman
}




\section{Background}

Dialysis is a successful therapeutic process used for removing metabolic waste from the body fluids of patients with end-stage renal disease (ESRD). There are two primary types of dialysis, namely peritoneal dialysis (PD) and hemodialysis [1]. More than one million ESRD people worldwide regularly undergo renal dialysis [1, 2]. However, microbial infection remains one of the most common complications among patients receiving renal dialysis [3]. According to a retrospective study of 327,993 renal dialysis patients conducted in the USA, chronic dialysis recipients have nearly 10 times the risk of fungal infections compared to the general population [4]. The cumulative annual incidence of infection-related hospitalization during renal dialysis was $26 \%$ for children and $31 \%$ for adults in the USA [5].

In addition to bacterial and viral infections, fungal infections are becoming increasingly frequent among renal dialysis patients, with significant morbidity and mortality rates [6]. For example, fungal infections account for an estimated 5\% of all PD-related peritonitis and have a higher mortality rate than bacterial PD-related peritonitis, with a mortality of 8.6-40.6\% [4]. Candida spp. (70\%) was the overwhelming microbial pathogen that caused fungal infections in patients receiving renal dialysis, followed by Cryptococcus spp. (6\%) and Coccidioides spp. (4\%) [4]. These data suggest that further attention should be paid to prevent or to timely diagnose fungal infections among ESRD patients during renal dialysis therapy.

The Cryptococcus genus, which belongs to the Basidiomycota, is the second most common group of fungal pathogens associated with renal dialysis patients [4, 7-9]. Cryptococcus neoformans and $C$. gattii are the main Cryptococcus species causing infections in humans. In contrast, Cryptococcus laurentii, Cryptococcus albidus and Cryptococcus arboriformis are generally regarded as saprophytes and have been rarely reported as agents causing human infections [10]. However, there has been an incremental rise in infections due to these organisms over recent decades [11]. This increase may reflect enhanced awareness of such infections, improved laboratory detection technology and a rise in the number of at-risk patients. These infections have a similar clinical presentation as those caused by $C$. neoformans or $C$. gattii, but cryptococcal antigen (CrAg) test results are frequently negative and the antifungal susceptibility patterns of these species are characterized by higher MICs [11, 12].

Despite the remarkable achievement of renal dialysis in prolonging the life of ESRD patients, the use of dialysis is problematic in some respects, such as fungal infection. Individual case reports of cryptococcosis in renal dialysis patients have been reported worldwide since 1980, and particularly since 2010. Currently, our understanding of cryptococcosis during renal dialysis is scarce and fragmented, which makes it challenging for physicians to timely diagnose and treat it among patients undergoing renal dialysis. Hence, we sought to perform a retrospective pooled analysis of the association between cryptococcosis and dialysis patients in our hospital and systematically reviewed the published reports, with a focus on epidemiology and clinical characteristics.

\section{Methods}

Case Collection

This study was conducted in Shanghai Changzheng hospital, a top renal dialysis center in Shanghai, China, which receives 85,000 renal dialysis cases annually. The protocol for this study was approved by the Institutional Review Board of Shanghai Changzheng Hospital (approval number 2016SL021). The cases included in the pooled analysis consisted of original cases from our hospital and published reports in electronic databases. The original cases were patients with a discharge diagnosis of "cryptococcosis" or "renal dialysis" who were admitted to our hospital between January 2001 and December 2016 and identified in the inpatient medical record database. We further systematically reviewed cases published in four major electronic literature databases: PubMed, Embase, ISI Web of Science, and Science Direct. The searches were limited to those written in English. The main search terms used were "cryptococcosis" and "dialysis," both as MeSH terms and free text words. A definitive diagnosis of cryptococcosis was defined as the identification of positive findings upon Cryptococcus culture, India ink staining, histology, or a $\mathrm{CrAg}$ test. 
Data Collection and Statistical Analysis

After the cases were identified, the following data were extracted for (1) demographic and epidemiological data, (2) clinical manifestations and laboratory test results, (3) mycological results, and (4) treatment strategies and outcomes. SPSS (version 21, International Business Machines Corporation, Armonk, NY, USA) and Graph Pad Prism (version 5, Graph Pad Software, Inc. La Jolla, CA, USA) were used for statistical analysis. Results are presented as the means \pm standard deviations (SDs) for normal data. A $P$ value of less than 0.05 was considered statistically significant.

\section{Results}

Case Review and Analysis

A total of 754 articles were identified in the initial screen of the four international databases. Of these articles, 735 were excluded because of duplication and/ or an inappropriate content. Nearly all of these pooled cases of cryptococcosis during renal dialysis were single case reports [13-27]. Among these, 16 cases reported in 15 articles with definitive diagnosis of cryptococcosis were analyzed further. In addition, we reported two renal dialysis cases with cryptococcosis who were hospitalized at our hospital. Consequently,

PubMed ( $\mathrm{n}=65)$; ISI Web of Science ( $\mathrm{n}=61)$; EMBASE $(\mathrm{n}=71)$; Science Direct $(\mathrm{n}=557)$

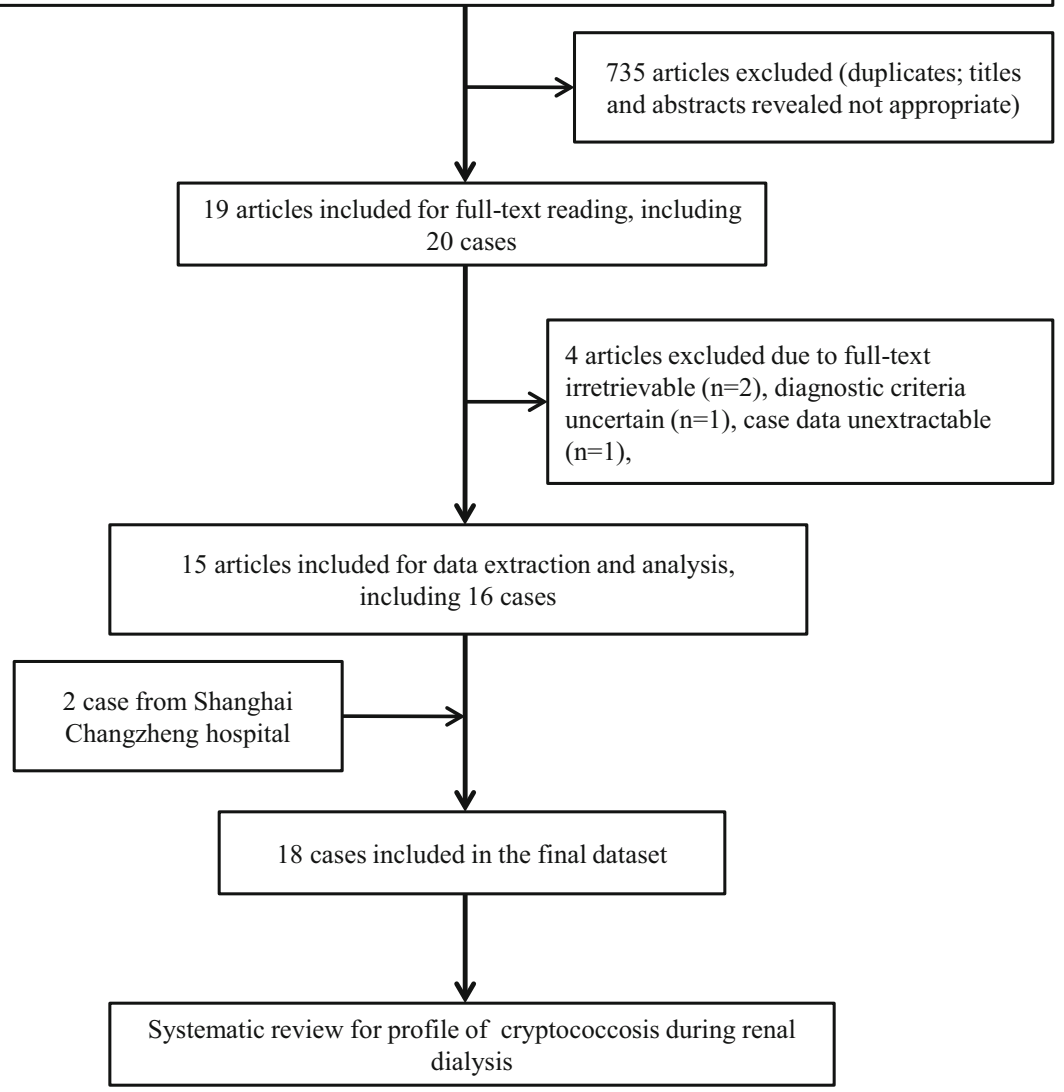

Fig. 1 Flow diagram of the search and review processes to identify cryptococcosis cases in renal dialysis patients 
18 cases were pooled for analysis in the current study. The details of these cases are provided in Fig. 1.

Demographic and Epidemiologic Characteristics

Among the 18 pooled cases, 10 cases were reported among PD patients and the remaining eight occurred among hemodialysis patients. The median duration of renal dialysis for PD and hemodialysis cases was 8 months (range 0.25-48 months) and 36 months (24-228 months), respectively. One PD-patient died because of cryptococcosis, and two patients with hemodialysis died because of cryptococcosis during renal dialysis therapy. All of the pooled patients were reported from medical centers in upper-middle to high-income countries (classification based on World Bank data: http://data.worldbank.org/about/countryand-lending- groups), with a male: female gender ratio of 13:5. Half of the pooled cases were reported since 2009. Most of the pooled cases were reported in the USA $(38.9 \%, 7 / 18)$, followed by Japan $(16.7 \%, 3 / 18)$, China $(16.7 \%, 2 / 18)$, UK $(16.7 \%, 2 / 18)$, Spain $(5.6 \%$, $1 / 18)$, Korea $(5.6 \%, 1 / 18)$, Brazil $(5.6 \%, 1 / 18)$, and New Zealand $(5.6 \%, 1 / 18)$. The age range containing most patients was 51-60 years $(27.8 \%, 5 / 18)$, and most patients were adults (aged $\geq 16$ years old; $88.9 \%, 16 / 18)$. Details of the demographic and epidemiologic characteristics of the pooled cases are provided in Table 1 and Fig. 2.

Clinical Manifestations

Among the 10 PD cases, most cryptococcal infections occurred in the abdomen ascites $(60 \%, 6 / 10)$, followed by two cases involving both the abdomen ascites and cerebrospinal fluid (CSF), one case involving the abdomen ascites and blood, and one case involving the abdomen ascites, blood and CSF. Fever (70.0\%, 7/10), cloudy dialysate $(70.0 \%, 7 / 10)$, and abdominal pain $(50.0 \%, 5 / 10)$ were the most common clinical features. Remarkably, bacterial peritonitis was diagnosed frequently $(30.0 \%, 3 / 10)$ in our pooled PD patients. The most common bacterial pathogens in this study were Aeromonas hydrophila, Staphylococcus epidermidis, and Streptococcus group D.

In contrast, the infection sites were variable among the 8 hemodialysis patients, including blood $(n=5)$, cervical lymphocytes $(n=1)$, pleural cavity $(n=2)$,

Table 1 Epidemiological characteristics of cryptococcosis during renal dialysis, 1985-2016

\begin{tabular}{|c|c|c|c|c|c|c|c|c|}
\hline Case & Date & Sex/age & Dialysis type & Geographical location & $\begin{array}{l}\text { Pigeon } \\
\text { contact }\end{array}$ & $\begin{array}{l}\text { Previous dialysis } \\
\text { duration (months) }\end{array}$ & Prognosis & References \\
\hline 1 & 1986 & $\mathrm{M} / 24$ & PD & Jacksonville, USA & ND & 12 & Cured & {$[18]$} \\
\hline 2 & May 1988 & $\mathrm{~F} / 16$ & PD & Little Rock, USA & ND & 0.25 & Cured & {$[20]$} \\
\hline 3 & May 1988 & $\mathrm{~F} / 49$ & PD & Little Rock, USA & ND & 0.5 & Died & {$[20]$} \\
\hline 4 & May 1989 & $\mathrm{M} / 52$ & $\mathrm{PD}$ & Manchester, UK & ND & 7 & Cured & [19] \\
\hline 5 & Nov 1989 & $\mathrm{~F} / 13$ & PD & Tampa, USA & ND & 12 & Cured & [16] \\
\hline 6 & 1992 & $\mathrm{~F} / 50$ & PD & Porirua, New Zealand & ND & 8 & Cured & {$[21]$} \\
\hline 7 & Oct 1993 & $\mathrm{M} / 48$ & PD & New York, USA & ND & 6 & Cured & {$[22]$} \\
\hline 8 & Mar 2014 & $\mathrm{M} / 57$ & PD & New York, USA & Neg & 48 & Cured & [14] \\
\hline 9 & Jul 2014 & $\mathrm{M} / 58$ & PD & Seoul, Korea & ND & 10 & Cured & [15] \\
\hline 10 & Apr 2015 & $\mathrm{M} / 32$ & PD & Osaka city, Japan & ND & 36 & Cured & {$[13]$} \\
\hline 11 & Aug 1985 & $\mathrm{M} / 22$ & Hemodialysis & Bristol, UK & ND & 24 & Cured & {$[23]$} \\
\hline 12 & Sep 1993 & $\mathrm{M} / 37$ & Hemodialysis & Philadelphia, USA & ND & 228 & Cured & [17] \\
\hline 13 & Aug 2009 & $\mathrm{~F} / 60$ & Hemodialysis & Curitiba, Brazil & ND & 48 & Died & [24] \\
\hline 14 & Jan 2009 & M/49 & Hemodialysis & Matsuyama, Japan & ND & 0.25 & Died & [27] \\
\hline 15 & Sep 2009 & $\mathrm{M} / 64$ & Hemodialysis & Okinawa, Japan & Pos & 60 & Cured & [26] \\
\hline 16 & Jun 2012 & $\mathrm{M} / 53$ & Hemodialysis & Oviedo, Spain & ND & 24 & Cured & {$[25]$} \\
\hline 17 & May 2012 & $\mathrm{M} / 36$ & Hemodialysis & Shanghai, China & $\mathrm{Neg}$ & 24 & Cured & This study \\
\hline 18 & Aug 2014 & M/45 & Hemodialysis & Shanghai, China & $\mathrm{Neg}$ & 96 & Cured & This study \\
\hline
\end{tabular}

$P D$ peritoneal dialysis, $M$ male, $F$ female, $N D$ no data 
pulmonary capillary embolism $(n=1)$, and skin $(n=1)$. Two patients were each infected at two body sites. Fever $(50.0 \%, 4 / 8)$ was the most common clinical feature, followed by headache $(25.0 \%, 2 / 8)$, cough $(25.0 \%, 2 / 8)$, pleural effusion $(25.0 \%, 2 / 8)$, cervical lymphadenopathy $(12.5 \%, 1 / 8)$, and

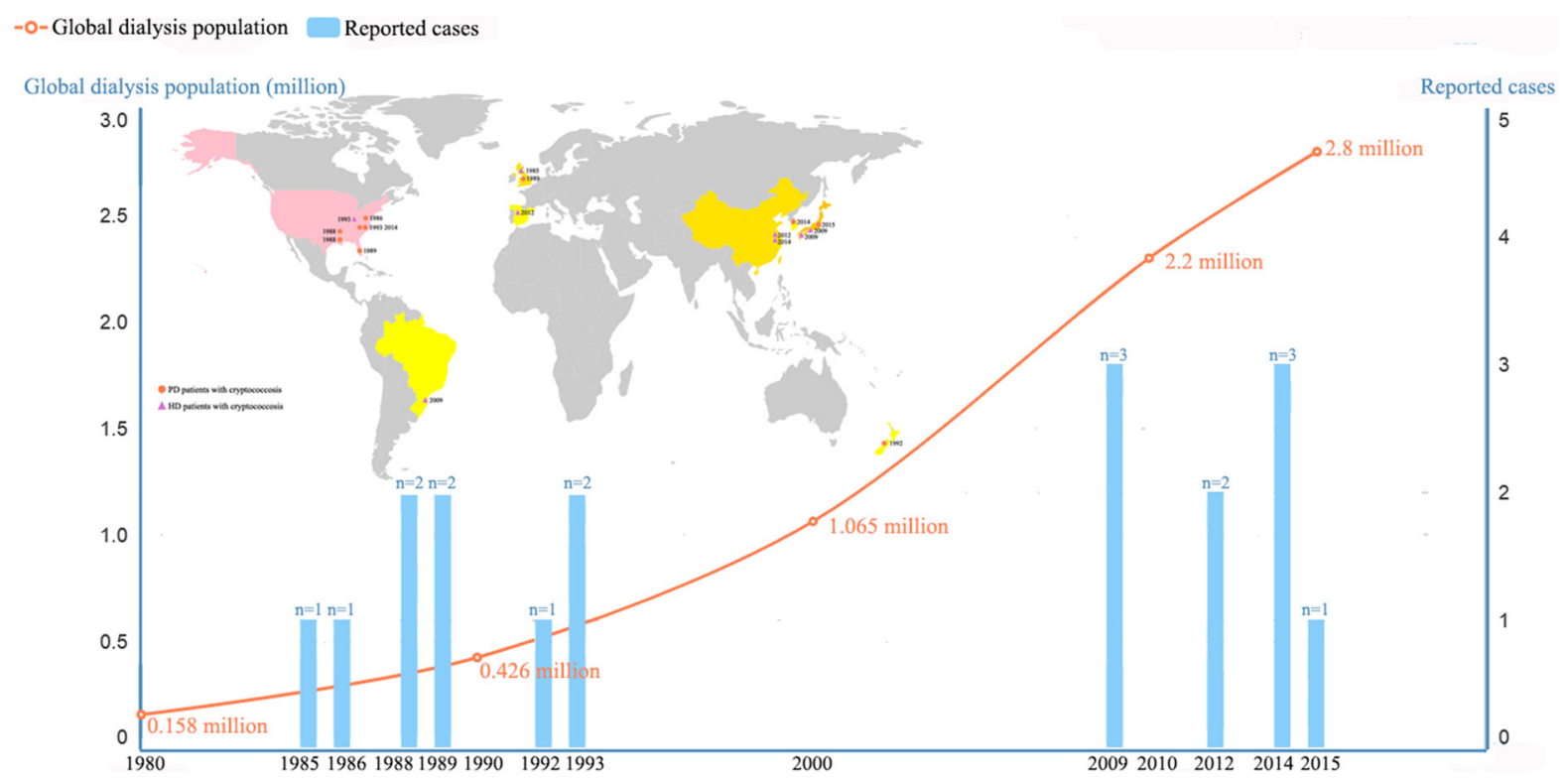

Fig. 2 Epidemiological profiles of renal dialysis patients with cryptococcosis in the present study, 1985-2016

Table 2 Clinical characteristics of cryptococcosis during renal dialysis

\begin{tabular}{|c|c|c|c|c|}
\hline Cases & Affected site & Main manifestation & Previous antibiotics & References \\
\hline 1 & Abdomen & Abdominal pain & Cefadyl & {$[18]$} \\
\hline 2 & Abdomen + blood + CSF & Fever & ND & {$[20]$} \\
\hline 3 & Abdomen + CSF & Abdominal pain + fever & ND & {$[20]$} \\
\hline 4 & Abdomen + blood & Cloudy dialysate + fever & ND & [19] \\
\hline 5 & Abdomen & Abdominal pain + cloudy dialysate + fever & Vancomycin & [16] \\
\hline 6 & Abdomen & cloudy dialysate & ND & {$[21]$} \\
\hline 7 & Abdomen + CSF & Cloudy dialysate + fever & Neg & {$[22]$} \\
\hline 8 & Abdomen & Abdominal pain + cloudy dialysate & Vancomycin & {$[14]$} \\
\hline 9 & Abdomen & Abdominal pain + cloudy dialysate + fever & Imipenem & {$[15]$} \\
\hline 10 & Abdomen & Cloudy dialysate + fever & Neg & [13] \\
\hline 11 & Cervical lymphocyte + blood & cervical lymphadenopathy + fever & ND & {$[23]$} \\
\hline 12 & Pleural cavity & Cough + fever & ND & {$[17]$} \\
\hline 13 & Blood & Fever & ND & {$[24]$} \\
\hline 14 & Pulmonary capillary embolism & Severe hypoxia + dyspnea & Vancomycin & {$[27]$} \\
\hline 15 & Pleural cavity & Cough & ND & {$[26]$} \\
\hline 16 & Skin + blood & Cutaneous ulceration & ND & {$[25]$} \\
\hline 17 & Blood + CSF & Headache & ND & This study \\
\hline 18 & Blood + CSF & Headache + fever & ND & This study \\
\hline
\end{tabular}

$C S F$ cerebrospinal fluid, $N D$ no data 

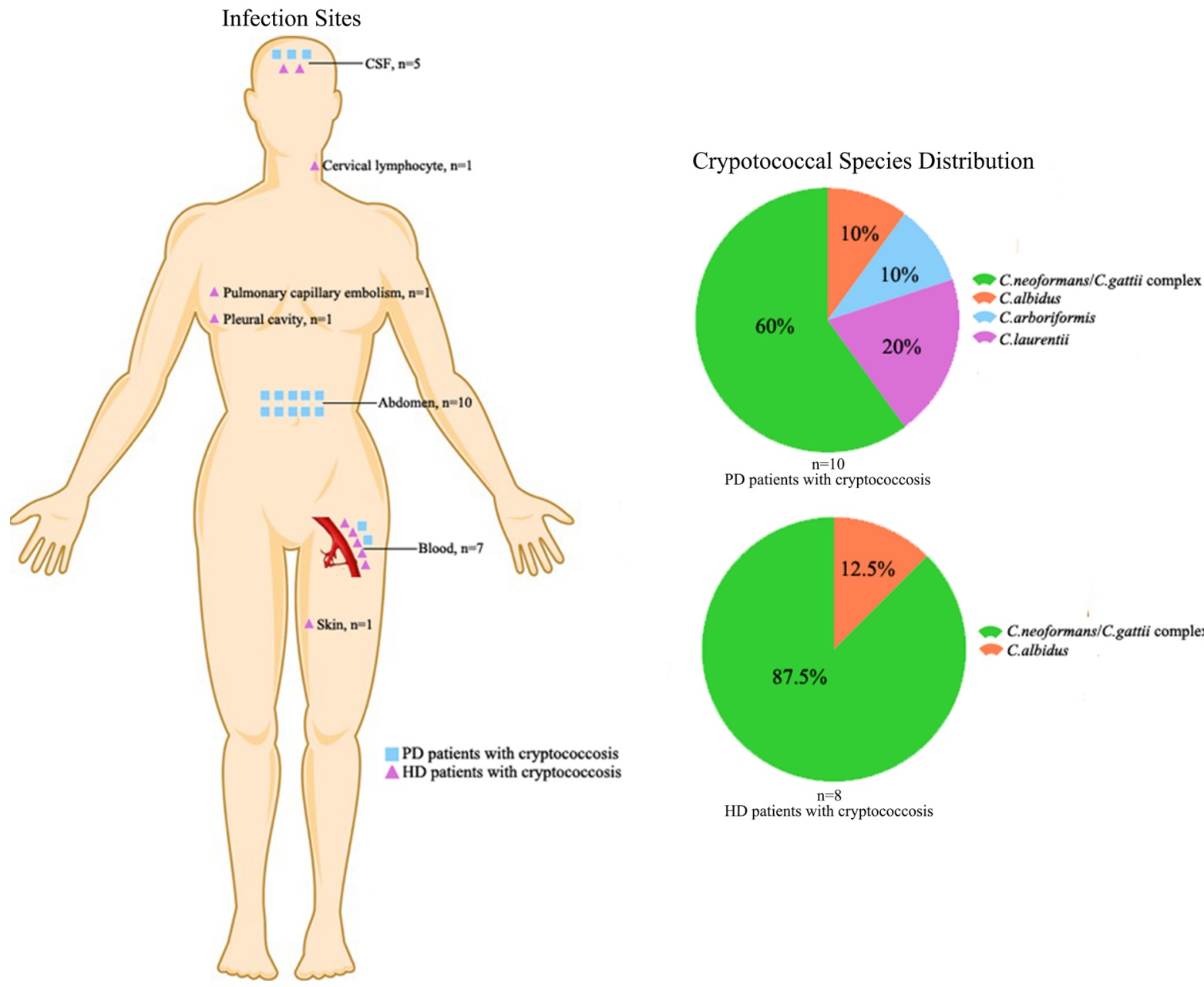

PD patients with cryptococcosis

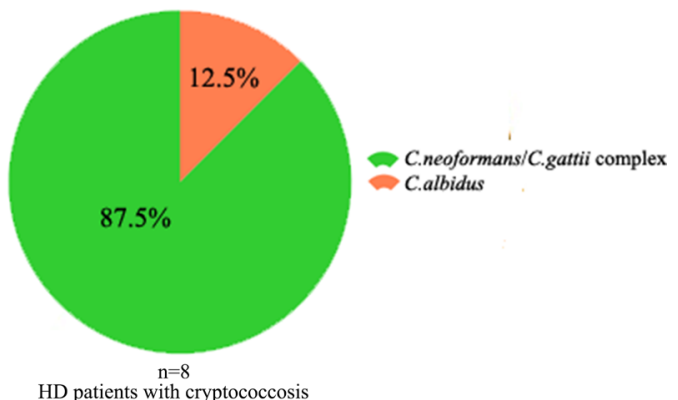

Fig. 3 Clinical characteristics and the related cryptococcus species in the pooled renal dialysis patients

erythematous lesions $(12.5 \%, 1 / 8)$. Details of the clinical manifestations are shown in Table 2 and Fig. 3.

\section{Diagnostic Profile}

Regarding the diagnostic profiles of the 18 pooled cases, 16 were diagnosed using cultures, 12 were diagnosed using $\mathrm{CrAg}$ tests, and two cases were diagnosed using India ink staining and histology. All cases $(100 \%, 4 / 4)$ caused by non- $C$. neoformans/C. gattii were CrAg-negative. Notably, multiple Cryptococcus species were observed in the 16 cases diagnosed using cultures, including $C$. neoformans (68.7\%, 11/16), C. laurentti (12.5\%, 2/16), C. albidus $(12.5 \%, 2 / 16)$, and C. arboriformis $(6.3 \%, 1 / 16)$. The details are provided in Table 3 .

\section{Treatment and Outcome}

The antifungal therapies used to treat the pooled cases were highly variable: amphotericin $\mathrm{B}(\mathrm{AmB})$ in combination with 5-fluorocytosine (5-FC) was used in seven cases, followed by $\mathrm{AmB}$ alone in six cases, $\mathrm{AmB}$ in combination with voriconazole (VCZ) in one case, $\mathrm{AmB}$ in combination with fluconazole (FCZ) in one case, $\mathrm{VCZ}$ alone in one case, $\mathrm{VCZ}$ in combination with 5-FC in one case, and ketoconazole (KCZ) in combination with FCZ in one case. In addition, catheters were removed from all the PD patients $(100 \%, 10 / 10)$ after the identification of cryptococcosis, whereas only one hemodialysis patient $(12.5 \%$, 1/8) had the catheter removed. The details are provided in Table 4. 
Table 3 Diagnosis profile of cryptococcosis during renal dialysis
Cr Ag cryptococcal antigen, $N D$ no data

\begin{tabular}{|c|c|c|c|}
\hline Cryptococcus species & Serum CrAg & Diagnostic evidence & References \\
\hline C. laurentii & Neg & Culture & {$[16]$} \\
\hline C. albidus & Neg & Culture & {$[14]$} \\
\hline C. arboriformis & $\mathrm{Neg}$ & Culture & {$[15]$} \\
\hline C. laurentii & Neg & Culture & {$[13]$} \\
\hline C. albidus & ND & Culture & {$[17]$} \\
\hline Cryptococcus spp. & ND & Culture & {$[18]$} \\
\hline C. neoformans/C. gattii complex & $>1: 12000$ & Culture & [19] \\
\hline C. neoformans/C.gattii complex & $1: 512$ & Culture & {$[20]$} \\
\hline C. neoformans/C. gattii complex & ND & Culture & {$[20]$} \\
\hline C. neoformans var. neoformans & ND & Culture & {$[21]$} \\
\hline C. neoformans/C. gattii complex & $1: 10000$ & Culture & {$[22]$} \\
\hline C. neoformans/C. gattii complex & Neg & Histology & {$[23]$} \\
\hline C. neoformans/C. gattii complex & ND & Culture & {$[24]$} \\
\hline C. neoformans var. neoformans & $1: 2048$ & Culture + histology & {$[25]$} \\
\hline C. neoformans/C. gattii complex & $1: 320$ & Culture & This study \\
\hline C. neoformans/C. gattii complex & $1: 5120$ & Culture & This study \\
\hline C. neoformans/C. gattii complex & Neg & Culture & {$[26]$} \\
\hline Cryptococcus spp. & ND & Microscopic examination & {$[27]$} \\
\hline
\end{tabular}

\section{Discussion}

Previous studies have shown that cryptococcosis can occur in HIV-negative patients [28, 29]. However, a detailed profile of cryptococcosis occurring after renal dialysis is difficult to determine. Recent studies suggested that non-C. neoformans/C. gattii species, which have higher MICs of current antifungal drugs, accounted for a large proportion of cryptococcal infections among renal dialysis patients [13-17]. Hence, cryptococcosis in renal dialysis patients should be further studied due to our poor understanding of this infection.

The current study showed that male adult patients were dominant among renal dialysis recipients with cryptococcosis, which is consistent with previous studies [30, 31]. A considerable number of pooled cases $(5 / 18)$ occurred in the past 5 years, which is likely to be related to the increasing number of renal dialysis patients worldwide [3]. Most of the current cases were collected from medical centers in major cities worldwide, including New York in the USA, Osaka in Japan, and Shanghai in China [13, 14, 22]. Thus, the availability of modern diagnostic tools might be relevant to the relatively high level of diagnosed cryptococcal infections in these cities. Although HIV infection is the most common underlying risk factor for cryptococcal infection [11, 12], none of the renal dialysis recipients with cryptococcosis were reported to be HIV-positive in the current study. However, we cannot neglect the risk of cryptococcosis among renal dialysis recipients who also have HIV infections.

The current survey found several different characteristics between PD and hemodialysis patients with cryptococcosis. For example, the duration of exposure to renal dialysis is directly related to the risk of septicemia [32], because a longer duration of renal dialysis may have a cumulative detrimental effect on immunity. In the current study, the median duration of hemodialysis was nearly threefold longer than that of PD cases. However, there were nearly twice as many reported cases of cryptococcosis in PD patients than hemodialysis patients, which indicated that peritoneal dialysis patients might have a higher risk for cryptococcosis compared with hemodialysis patients. Remarkably, cryptococcal infections tend to be localized in the abdomen of PD patients $(60.0 \%, 6 / 10)$. In contrast, the sites of cryptococcal infection vary among hemodialysis patients, including skin, lungs, and blood. Moreover, the current data suggest that 
Table 4 Treatment profile of cryptococcosis during renal dialysis

\begin{tabular}{|c|c|c|c|c|}
\hline Case & $\begin{array}{l}\text { Removal of } \\
\text { catheter }\end{array}$ & Antifungal treatment & Outcome & References \\
\hline 1 & Yes & AmB (cumulative dose, $0.3 \mathrm{~g}$ ) & $\begin{array}{l}\text { Switching to permanent } \\
\text { hemodialysis }\end{array}$ & {$[18]$} \\
\hline 2 & Yes & AmB (cumulative dose, $2 \mathrm{~g}$ ) & Cured & {$[20]$} \\
\hline 3 & Yes & $\mathrm{AmB}+5-\mathrm{FC}$ & Died & {$[20]$} \\
\hline 4 & Yes & AmB (cumulative dose, $1.4 \mathrm{~g}$ ) +5 -FC (cumulative dose, $55 \mathrm{~g}$ ) & $\begin{array}{l}\text { Switching to permanent } \\
\text { hemodialysis }\end{array}$ & [19] \\
\hline 5 & Yes & $\mathrm{MCZ} \times 3$ days $+\mathrm{AmB}$ (cumulative dose, $0.5 \mathrm{~g}$ ) & Cured & [16] \\
\hline 6 & Yes & $\mathrm{KCZ} 400 \mathrm{mg} /$ day $\times 2$ days $+\mathrm{FCZ} 400 \mathrm{mg} /$ day $\times 5$ days & Cured & {$[21]$} \\
\hline 7 & Yes & $\mathrm{AmB} 0.5 \mathrm{mg} / \mathrm{kg} /$ day $\times 6$ weeks +5 -FC $\times 4$ weeks & $\begin{array}{l}\text { Switching to permanent } \\
\text { hemodialysis }\end{array}$ & {$[22]$} \\
\hline 8 & Yes & $\begin{array}{l}(\text { FCZ } 200 \mathrm{mg} / \text { day }+ \text { CPF } 50 \mathrm{mg} / \text { day }) \times 5 \text { days }+ \text { AmB } \\
\text { liposomal } 400 \mathrm{mg} / \text { day } \times 7 \text { days }\end{array}$ & $\begin{array}{l}\text { Switching to permanent } \\
\text { hemodialysis }\end{array}$ & [14] \\
\hline 9 & Yes & FCZ200 mg/day $\times 6$ days + AmB $0.5 \mathrm{mg} / \mathrm{kg} /$ day $\times 4$ weeks & $\begin{array}{l}\text { Switching to permanent } \\
\text { hemodialysis }\end{array}$ & {$[15]$} \\
\hline 10 & Yes & VCZ $500 \mathrm{mg} \times 8$ days & Cured & [13] \\
\hline 11 & No & AmB (cumulative dose, $2.5 \mathrm{~g}$ ) $+5-\mathrm{FC} 50 \mathrm{mg} / \mathrm{kg} \times 6$ weeks & Cured & [23] \\
\hline 12 & No & AmB (cumulative dose, $1.9 \mathrm{~g}$ ) & Cured & [17] \\
\hline 13 & Yes & $\mathrm{AmB} 0.5 \mathrm{mg} / \mathrm{kg} / \mathrm{day} \times 6$ weeks & Died & {$[24]$} \\
\hline 14 & ND & ND & Died & [27] \\
\hline 15 & No & $(\mathrm{AmB}+5-\mathrm{FC}) \times 9$ days $+\mathrm{FCZ} \times 24$ weeks & Cured & {$[26]$} \\
\hline 16 & No & $\mathrm{VCZ} 400 \mathrm{mg} / \mathrm{d} \times 10$ days $+\mathrm{AmB} 100 \mathrm{mg} /$ day $\times 4$ days + & Cured & [25] \\
\hline 17 & No & $(\mathrm{VCZ} 0.4 \mathrm{~g} /$ day +5 -FC $4 \mathrm{~g} /$ day $) \times 2$ weeks & Cured & This study \\
\hline 18 & No & $($ AmB $30 \mathrm{mg} /$ days +5 -FC $4.5 \mathrm{~g} /$ day $) \times 4$ weeks & Cured & This study \\
\hline
\end{tabular}

$A m B$ Amphotericin B, $V C Z$ voriconazole, $F C Z$ fluconazole, $K C Z$ ketoconazole, $M C Z$ miconazole, 5-FC 5-fluorocytosine, $C P F$ caspofungin, $N D$ no data

several PD patients $(40.0 \%, 4 / 10)$ had previously been treated for bacterial peritonitis. This finding is consistent with a study [33] encompassing 66 centers in Australia over a 4-year period, which indicated that previously treated bacterial peritonitis is a major risk factor for fungal peritonitis.

Cryptococcus neoformans and C. gattii species are responsible for almost all human cryptococcal infections [7]. Other cryptococcal species were traditionally considered to be nonpathogenic [11, 12]. However, cases of cryptococcosis caused by nonC. neoformans/C. gattii have increased significantly since the 1970s, including in renal dialysis patients. The results of the current study also revealed that nonneoformans/gattii cryptococcal species account for a considerable proportion (approximately 27.8\%, 5/18) of the cryptococcal species among renal dialysis patients [13-17], particularly $C$. laurentii or $C$. albidus. This observation is consistent with a recent global review of 44 previously published cases [11]. Notably, the current results suggest that CrAg tests are ineffective for the diagnosis of cryptococcosis caused by non-C. neoformans/C. gattii species, although this test is considered as a powerful tool for the diagnosis of cryptococcal infections caused by C. neoformans or C. gattii species [34-36]. The discrepancy in the sensitivity of $\mathrm{CrAg}$ test might be related to the significant phylogenetic divergence between nonC. neoformans/C. gattii species and C. neoformans/ C. gattii species complex [8, 9]. Indeed, recent phylogenetic studies have reclassified $C$. laurentii to Papiliotrema laurentii, C. albidus to Naganishia albida and C. arboriformis to Cutaneotrichosporon arboriformis [8, 9]. Another potential cause of the negative $\mathrm{CrAg}$ test for infections caused by $C$. laurentii and $C$. albidus might be related to a lower organism burden in cryptococcemia caused by these non- $C$. neoformans/C. gattii species [11]. Thus, a 
negative $\mathrm{CrAg}$ test result cannot rule out cryptococcal infections caused by non- $C$. neoformans/C. gattii species [37].

Regarding the treatment of cryptococcosis among dialysis patients in this study, the catheter was removed from most PD patients (91\%) after the identification of a cryptococcal infection. This is consistent with a recent retrospective review indicating that catheters should be removed immediately and certainly within $24 \mathrm{~h}$ after the identification of fungi in PD patients with fungal peritonitis [38]. In contrast, only one hemodialysis patient had the catheter removed. Moreover, it has been shown that the risks of recurrent fungal peritonitis and death were lowest when the catheter removal is combined with antifungal therapy compared with either intervention alone [33]. In addition, multiple antifungal agents were used to treat cryptococcosis patients with renal dialysis in the current study, including AmB, FCZ, VCZ, KCZ, and flucytosine. Among these, AmB is the most frequently used antifungal drug, which is consistent with the 2010 guidelines for the treatment of cryptococcosis [39]. Treatment with AmB and FCZ is the previous recommendations for cryptococcal infections caused by nonC. neoformans/C. gattii species, including C. albidus, C. laurentii, C. uniguttulatus and C. curvatus, according to the results of an in vitro susceptibility study [12]. In addition, recent susceptibility testing of $C$. arboriformis revealed that the isolate was susceptible to AmB and FCZ, with intermediate sensitivity to flucytosine [15]. Further studies on renal dialysis recipients with cryptococcosis are needed to develop more effective and consistent treatment strategies.

In conclusion, cryptococcosis is a neglected complication among renal dialysis recipients and has shown an increasing tendency in recent years. Multiple species, such as $C$. albidus, $C$. laurentii and $C$. arboriformis, were observed with nonspecific clinical manifestations. Physicians should consider the possibility of cryptococcosis among renal dialysis recipients, even for those with a negative CrAg test result. The timely removal of the catheter was shown to be crucial for the successful recovery of PD patients with cryptococcosis. Optimizing antifungal treatment strategy in renal dialysis recipients with cryptococcal infections is needed.

Acknowledgements This study was funded in part by grants from the 973 Program (2013CB531601 and 2013CB531606),
Major National R\&D Projects of the National Health Department (2014ZX09J14106-02A), National Natural Science Foundation of China (81201269), and Shanghai Science and Technology Committee (Grant number 14DZ2272900), Second Military Medical University (2017JZ49).

\section{Compliance with Ethical Standards}

Conflict of interest statement The authors declared that they have no conflicts of interest.

Open Access This article is distributed under the terms of the Creative Commons Attribution 4.0 International License (http:// creativecommons.org/licenses/by/4.0/), which permits unrestricted use, distribution, and reproduction in any medium, provided you give appropriate credit to the original author(s) and the source, provide a link to the Creative Commons license, and indicate if changes were made.

\section{References}

1. Himmelfarb J, Ikizler TA. Hemodialysis. New Engl J Med. 2010;363(19):1833-45. doi:10.1056/NEJMra0902710.

2. Ori Y, Korzets A, Malachi T, Gafter U, Breitbart $H$. Impaired lymphocyte calcium metabolism in end-stage renal disease: enhanced influx, decreased efflux, and reduced response to mitogen. J Lab Clin Med. 1999;133(4): $391-400$.

3. Li PK, Chow KM. Infectious complications in dialysisepidemiology and outcomes. Nat Rev Nephrol. 2011;8(2):77-88. doi:10.1038/nrneph.2011.194.

4. Abbott KC, Hypolite I, Tveit DJ, Hshieh P, Cruess D, Agodoa LY. Hospitalizations for fungal infections after initiation of chronic dialysis in the United States. Nephron. 2001;89(4):426-32.

5. O'Riordan E, Conlon PJ. Haemodialysis catheter bacteraemia: evolving strategies. Curr Opin Nephrol Hypertens. 1998;7(6):639-42.

6. Johnson DW, Dent H, Hawley CM, McDonald SP, Rosman JB, Brown FG, et al. Associations of dialysis modality and infectious mortality in incident dialysis patients in Australia and New Zealand. Am J Kidney Dis. 2009;53(2):290-7. doi:10.1053/j.ajkd.2008.06.032.

7. Fonseca A, Fell J, Boekhout T. Cryptococcus Vuillemin (1901). In: Kurtzman C, Fell J, Boekhout T, editors. The yeasts. 5th ed. Amsterdam: Elsevier; 2011. p. 1661-737.

8. Liu XZ, Wang QM, Theelen B, Groenewald M, Bai FY, Boekhout T. Phylogeny of tremellomycetous yeasts and related dimorphic and filamentous basidiomycetes reconstructed from multiple gene sequence analyses. Stud Mycol. 2015;81:1-26. doi:10.1016/j.simyco.2015.08.001.

9. Liu XZ, Wang QM, Goker M, Groenewald M, Kachalkin $\mathrm{AV}$, Lumbsch HT, et al. Towards an integrated phylogenetic classification of the tremellomycetes. Stud Mycol. 2015;81:85-147. doi:10.1016/j.simyco.2015.12.001.

10. Bernal-Martinez L, Gomez-Lopez A, Castelli MV, MesaArango AC, Zaragoza O, Rodriguez-Tudela JL, et al. Susceptibility profile of clinical isolates of non-Cryptococcus 
neoformans/non-Cryptococcus gattii Cryptococcus species and literature review. Med Mycol. 2010;48(1):90-6. doi:10. 3109/13693780902756073.

11. Khawcharoenporn T, Apisarnthanarak A, Mundy LM. Nonneoformans cryptococcal infections: a systematic review. Infection. 2007;35(2):51-8. doi:10.1007/s15010-007-6142-8.

12. Arendrup MC, Boekhout T, Akova M, Meis JF, Cornely OA, Lortholary O. ESCMID and ECMM joint clinical guidelines for the diagnosis and management of rare invasive yeast infections. Clin Microbiol Infect. 2014;20(Suppl 3):76-98.

13. Asano M, Mizutani M, Nagahara Y, Inagaki K, Kariya T, Masamoto D, et al. Successful treatment of Cryptococcus laurentii peritonitis in a patient on peritoneal dialysis. Intern Med (Tokyo, Japan). 2015;54(8):941-4. doi:10.2169/ internalmedicine.54.3586.

14. Ragupathi L, Reyna M. Case report of Cryptococcus albidus peritonitis in a peritoneal dialysis patient and a review of the literature. Perit Dial Int. 2015;35(4):421-7.

15. Im H, Chae JD, Yoo M, Lee SY, Song EJ, Sung SA, et al. First case of continuous ambulatory peritoneal dialysis-related peritonitis caused by Cryptococcus arboriformis. Ann Lab Med. 2014;34(4):328-31.

16. Sinnott JT, Rodnite J, Emmanuel PJ, Campos A. Cryptococcus laurentii infection complicating peritoneal dialysis. Pediatr Infect Dis J. 1989;8(11):803-5.

17. Horowitz ID, Blumberg EA, Krevolin L. Cryptococcus albidus and mucormycosis empyema in a patient receiving hemodialysis. South Med J. 1993;86(9):1070-2.

18. Morford DW, Sandroni SE. Cryptococcal peritonitis review. In: Advances in peritoneal dialysis, vol 2 1986, Part Four. International Society for Peritoneal Dialysis. 2016. http://www.advancesinpd.com/. Accessed 10 Sept 2016.

19. Kaczmarski EB, Tooth JA, Anastassiades E, Manos J, Gokal R. Cryptococcosis complicating continuous ambulatory peritoneal dialysis. J Infect. 1989;18(3):289-92.

20. Smith JW, Arnold WC. Cryptococcal peritonitis in patients on peritoneal dialysis. Am J Kidney Dis. 1988;11(5):430-3.

21. Morris B, Chan YF, Reddy J, Woodgyer A. Cryptococcal peritonitis in a CAPD patient. J Med Vet Mycol. 1992;30(4):309-15.

22. Yinnon AM, Solages A, Treanor JJ. Cryptococcal peritonitis: report of a case developing during continuous ambulatory peritoneal dialysis and review of the literature. Clin Infect Dis. 1993;17(4):736-41.

23. Banks R, Williams A, Glover S, Burton P, Warnock D, Mackenzie C. Disseminated cryptococcosis in a patient receiving chronic haemodialysis. Postgrad Med J. 1985;61(718):745-7.

24. Tuon FF, Morales HM, Penteado-Filho SR, da-Silva MM, Quadros I, El Hamoui A. Central venous catheter-related bloodstream infection and Cryptococcus neoformans. Braz J Infect Dis. 2009;13(4):317-8.

25. Latino GA, Gago E, Vidau P, Vivanco B. Cutaneous cryptococcosis in a patient on chronic haemodialysis. Nefrologia. 2012;32(5):697-8.

26. Kinjo K, Satake S, Ohama T. Cryptococcal pleuritis developing in a patient on regular hemodialysis. Clin Nephrol. 2009;72(3):229-33.
27. Eriguchi M, Nagao T, Kamimura T, Sugawara K, Mitsuiki K, Harada A. Pulmonary capillary embolism caused by cryptococcemia in a hemodialysis patient. Clin Nephrol. 2009;71(1):88-91.

28. Yuchong C, Fubin C, Jianghan C, Fenglian W, Nan X, Minghui Y, et al. Cryptococcosis in China (1985-2010): review of cases from Chinese database. Mycopathologia. 2012;173(5-6):329-35. doi:10.1007/s11046-011-9471-1.

29. Tseng HK, Liu CP, Ho MW, Lu PL, Lo HJ, Lin YH, et al. Microbiological, epidemiological, and clinical characteristics and outcomes of patients with cryptococcosis in Taiwan, 1997-2010. PLoS ONE. 2013;8(4):e61921. doi:10. 1371/journal.pone.0061921.

30. Chen M, Al-Hatmi AM, Chen Y, Ying Y, Fang W, Xu J, et al. Cryptococcosis and tuberculosis co-infection in mainland China. Emerg Microbes Infect. 2016;5(9):e98.

31. Fang W, Hong N, Li Y, Liu J, Zhang L, Jiang W, et al. Cryptococcosis in patients with nephrotic syndrome: a pooled analysis of cases. Mycopathologia. 2017; doi:10. 1007/s11046-016-0101-9.

32. Powe NR, Jaar B, Furth SL, Hermann J, Briggs W. Septicemia in dialysis patients: incidence, risk factors, and prognosis. Kidney Int. 1999;55(3):1081-90. doi:10.1046/j. 1523-1755.1999.0550031081.x.

33. Miles R, Hawley CM, McDonald SP, Brown FG, Rosman $\mathrm{JB}$, Wiggins KJ, et al. Predictors and outcomes of fungal peritonitis in peritoneal dialysis patients. Kidney Int. 2009;76(6):622-8. doi:10.1038/ki.2009.202.

34. Boulware DR, Rolfes MA, Rajasingham R, von Hohenberg M, Qin Z, Taseera K, et al. Multisite validation of cryptococcal antigen lateral flow assay and quantification by laser thermal contrast. Emerg Infect Dis. 2014;20(1):45-53. doi:10.3201/eid2001.130906.

35. Tang MW, Clemons KV, Katzenstein DA, Stevens DA. The cryptococcal antigen lateral flow assay: a point-of-care diagnostic at an opportune time. Crit Rev Microbiol. 2016;42(4):634-42. doi:10.3109/1040841x.2014.982509.

36. Chen M, Zhou J, Li J, Li M, Sun J, Fang WJ, et al. Evaluation of five conventional and molecular approaches for diagnosis of cryptococcal meningitis in non-HIV-infected patients. Mycoses. 2016;59(8):494-502. doi:10.1111/myc. 12497.

37. Ramchandren R, Gladstone DE. Cryptococcus albidus infection in a patient undergoing autologous progenitor cell transplant. Transplantation. 2004;77(6):956.

38. Chang TI, Kim HW, Park JT, Lee DH, Lee JH, Yoo TH, et al. Early catheter removal improves patient survival in peritoneal dialysis patients with fungal peritonitis: results of ninety-four episodes of fungal peritonitis at a single center. Perit Dial Int. 2011;31(1):60-6.

39. Perfect JR, Dismukes WE, Dromer F, Goldman DL, Graybill JR, Hamill RJ, et al. Clinical practice guidelines for the management of cryptococcal disease: 2010 update by the infectious diseases society of america. Clin Infect Dis. 2010;50(3):291-322. 